\title{
Extraction of aluminium surface energies from slab calculations: perturbative and non-perturbative approaches
}

\author{
Carlos Fiolhais $^{\text {a,* }}$, L.M. Almeida ${ }^{\text {b,c }}$, C. Henriques ${ }^{\text {d }}$

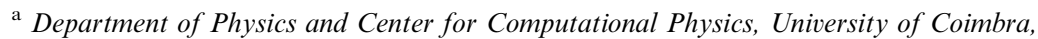 \\ P-3004-516 Coimbra, Portugal \\ b Department of Physics, University of Aveiro, P-3810 Aveiro, Portugal \\ ${ }^{\mathrm{c}}$ Center for Computational Physics, University of Coimbra, P-3004-516 Coimbra, Portugal \\ ${ }^{\mathrm{d}}$ Department of Physics, School of Sciences and Technology, New University of Lisbon, \\ P-2825-114 Caparica, Portugal
}

\begin{abstract}
Surface energies of aluminium ((llll 111$),\left(\begin{array}{lll}1 & 1 & 0\end{array}\right)$ and $\left.\left(\begin{array}{lll}1 & 0 & 0\end{array}\right)\right)$ were calculated in second-order perturbation theory based on the jellium model, and by full atomistic models using a Gaussian basis set, in the framework of density functional theory. In both cases, surface energies were extracted from slab calculations using the incremental method, which considers two slabs with consecutive numbers of layers (6 and 7 layers). In the non-perturbative calculation, the fitting method which involves a series of slabs up to 10 layers is also used to examine the limitations of the incremental method and to improve it. Our results are compared with those from other authors and with experiment being the limitations of the perturbative method discussed. The predictions of the stabilized jellium model are also referred to.
\end{abstract}

(C) 2003 Elsevier Ltd. All rights reserved.

Keywords: Surface energy; Slabs; Density-functionals; Perturbative method; All-electron; Jellium model

\section{Introduction}

The surface energy is, by definition, half of the energy necessary to break the bulk solid in two pieces. The calculation of the surface energy of a given material may be

\footnotetext{
*Corresponding author. Tel.: +351-239-410624; fax: +351-239-829158.

E-mail addresses: tcarlos@teor.fis.uc.pt (C. Fiolhais), lma@fis.ua.pt (L.M. Almeida), crh@fct.unl.pt (C. Henriques).

$U R L:$ http://nautilus.fis.uc.pt/personal/ cfiolhais 
performed in various ways. The extraction of the surface energy from a series of slabs with different thickness turns out to be easier than the calculation of the semiinfinite system. The extrapolation to the semi-infinite system from thin slabs is necessary, since it is impracticable to evaluate the energy of a very thick slab. The main drawback of these thin slab calculations is the presence of important quantumsize effects, i.e., energy fluctuations induced by the finite size of the slab [1].

The energy of a slab with $N$ layers can be written as the sum of a contribution from the bulk, which is proportional to the volume $V$, and another from the surface, which is proportional to area $S$, whence:

$$
E_{\text {slab }}=\epsilon_{\text {bulk }} V+2 \sigma S,
$$

where $\epsilon_{\text {bulk }}$ is the energy density of the bulk crystal and $\sigma$ the surface energy. From this equation, we obtain

$$
\sigma=\frac{1}{2 S}\left(E_{\text {slab }}-\epsilon_{\text {bulk }} V\right) .
$$

The surface energy is small compared with the two terms from which it is calculated. Therefore, it is expected to be numerically very sensitive to the precision of those two terms.

Boettger [2] pointed out that the right-hand side of (2) diverges linearly with the number of layers in the slab. This fact is due to the separate calculation of the bulk energy density $\epsilon_{\text {bulk }}$, so that the bulk term in the energy of the slab is not exactly canceled by the energy of a bulk piece of the same size.

To avoid an independent calculation of the bulk energy, various methods for extracting the surface energy from slab energies have been proposed [2-5]. Two possibilities are considered in this work:

(i) taking the difference between the energies of two slabs;

(ii) and fitting the series of energies of several slabs.

In method (i), the bulk energy is approximated [2] by the difference between the energy of two slabs, for instance, one with $N$ and the other with $N+1$ atomic layers. In this so-called incremental energy approximation, we obtain an approximation $\epsilon_{\text {bulk }}^{*}$ to the bulk energy density, viz.,

$$
\frac{E_{\text {slab }}(N+1)}{S}-\frac{E_{\text {slab }}(N)}{S}=d \epsilon_{\text {bulk }}^{*}
$$

where $d$ is the thickness of a single layer. We rewrite (2), taking a slab with $N+1$ layers and $\epsilon_{\text {bulk }}^{*}$ instead of $\epsilon_{\text {bulk }}$, the result being

$$
\sigma=\frac{1}{2}\left[\frac{E_{\text {slab }}(N+1)}{S}-\epsilon_{\text {bulk }}^{*}(N+1) d\right] .
$$

Combining (4) and (3), the surface energy becomes

$$
\sigma=\frac{1}{2 S}\left[-N E_{\text {slab }}(N+1)+(N+1) E_{\text {slab }}(N)\right]
$$


which can be easily implemented, since it only requires the energies of two slabs. However, the oscillatory nature of the quantum size effects, which in some cases persist even for considerable thick slabs [1], may cast doubts on the stability of the result when $N$ changes.

In contrast, method (ii) [1,6] is more stable. Considering a slab with $N$ layers, (1) may be written as

$$
\frac{E_{\text {slab }}(N)}{S}=\epsilon_{\text {bulk }} d N+2 \sigma .
$$

Using a series of slabs with different $N$, it is possible to fit the parameters $\epsilon_{\text {bulk }}$ and $\sigma$. This is called the linear fitting method. We note that the incremental energy method is equivalent to a linear fitting with the energies of just two slabs.

The slab energies $E_{\text {slab }}$ are usually calculated solving the Kohn-Sham (KS) equations of density functional theory [7]. The only approximation in the KS equations is the density functional chosen to calculate the exchange and correlation energy. The simplest approximation is the local density approximation (LDA), which is based on the physics of the uniform electron gas [7]. Other approximations have been proposed to improve the LDA. Corrections using density gradients (generalized gradient approximations, GGA) are very popular. One of the most successful GGA is that proposed by Perdew, Burke and Ernzerhof (PBE) [8], which proved successful in many calculations, although the improvement of GGA with respect to LDA is limited in solid-state calculations [9]. A new generation of approximations, which goes under the name of Meta-GGA (namely, the PKZB [10], due to Perdew and co-workers), is nowadays being offered.

The jellium model, where the ions are replaced by a uniform positive background, is a good benchmark for testing functional approximations. LDA-KS results of surface energies for the jellium model using a semi-infinite system [11] are rather close to results that are supposed to be almost exact, namely, a refinement of the RPA [12] called RPA+ [13]. On the other hand, GGA-PBE surface energies are generally lower [14]. This paradox has been discussed by Almeida et al. [15]. MetaGGA-PKZB has been employed to improve the GGA surface energy of the jellium model [14].

In this paper, two types of density-functional calculations are presented for the surface energies of aluminium. One is perturbative, relying on the jellium model, and the other is non-perturbative. Both use slabs with unrelaxed ionic planes (faces (1 111$),\left(\begin{array}{lll}1 & 1 & 0\end{array}\right)$ and $\left.\left(\begin{array}{lll}1 & 0 & 0\end{array}\right)\right)$. According to Ho and Bohnen [16], the effect of multilayer relaxation in aluminium surface energies is small $(<2 \%)$.

We start by presenting second-order perturbative corrections to the surface energy of a jellium, which are due to the ionic structure of the real metal [17-19]. The core potentials are described by a local pseudopotential, such as the local form of the Heine-Abarenkov (HA) [20,21], i.e., independent of the angular momentum. The potential depth is $u=-0.3321$ and the core radius $R_{\mathrm{c}}=1.4017$, both in atomic units. Since the pseudopotential is weak, perturbation theory based on jellium works for the bulk [22] and should work reasonably well for the surface of simple metals. 
The first-order perturbative correction to the surface energy is well-known [11]. For aluminum, it is large enough to make positive the surface energy, which is negative in the jellium model.

The second-order term, which should be added to the former, was evaluated with the incremental energy method indicated by (5). This correction to the surface energy of a 7 layer slab was obtained from the energies of slabs with 6 and 7 layers,

$$
\sigma^{(2)}=\frac{1}{S}\left[-6 E_{\mathrm{ps}}^{(2)}(7)+7 E_{\mathrm{ps}}^{(2)}(6)\right]
$$

We have also performed ab-initio non-perturbative calculations considering full potentials, which are, in principle, more accurate. Our all-electron calculations required Gaussians functions as the basis to describe the KS orbitals [23]. The surface energies of aluminium were extracted, using not only the incremental method, but also the more reliable linear fitting method (6), which in our calculations included a series of slabs going from 1 to 10 layers.

We adopted a method similar to that of Fiorentini and Methfessel [3]. By checking the convergence of the surface energy, with respect to the number of slabs included in the fitting, we verified the better accuracy of this method relatively to the incremental method.

More details on the non-perturbative and perturbative methods are given in the following section. In Section 3, we present and discuss our results. The conclusions, namely, the comparisons between the incremental and the fitting methods, appear in Section 4.

\section{Calculation methods}

In the second-order perturbative correction to the jellium model [24], the electronic density of a slab changes, due to the linear response of the difference between the sum of ion pseudopotentials and the jellium background potential, i.e.,

$$
\delta V_{\mathrm{pp}}=V_{\mathrm{pp}}-V_{\mathrm{jellium}}
$$

The second-order energy of a slab is given by

$$
E^{(2)}=\frac{1}{2 S} \sum_{\vec{G}_{\|}} \int_{0}^{L} \int_{0}^{L} \delta V_{\mathrm{ps}}\left(-\vec{G}_{\|} \mid z\right) \chi^{S}\left(\vec{G}_{\|} \mid z, z^{\prime}\right) \delta V_{\mathrm{ps}}\left(\vec{G}_{\|} \mid z^{\prime}\right) \mathrm{d} z^{\prime} \mathrm{d} z,
$$

where $L$ is the width of a box containing the jellium electronic density, $z$ the coordinate perpendicular to the surface, and $\chi^{S}$ the linear response function obtained in the RPA [12]. All quantities inside the integrals are 2D Fourier transformed, $\vec{G}_{\|}$ being surface reciprocal lattice vectors. Details of the calculations can be found in $[17,19]$. We used the LDA parametrization for the correlation energy in the form proposed by Perdew and Wang [25]. 
On the other hand, we performed atomistic calculations of surface energies using the CRYSTAL98 program package [26], which implements the KS equations with full potentials using Gaussians as basis set.

The exponents of the most outer shells of the basis sets, which have been calculated in a previous work for the bulk system [27], were reoptimized using a slab with three layers and taking the $\left[\begin{array}{lll}1 & 0 & 0\end{array}\right]$ surface as reference. The convergence with respect to number of $k$-points in the Brillouin zone was checked. In order to achieve better convergence, a temperature smearing of 0.001 hartree of the Fermi surface was done. We used the same LDA formula as in the perturbative case.

\section{Results}

Table 1 shows our surface energies results for three different surfaces of aluminium (fcc), corresponding to (1 111$),\left(\begin{array}{lll}1 & 0 & 0\end{array}\right)$ and (1 110$)$ Miller indexes, compared with those obtained by other authors. We take for the lattice parameter the experimental value $4.05 \AA$.

Our second-order perturbation results appear in column 2 (HA pseudopotential) of Table 1 . There are only a few second-order perturbation calculations to compare with; one of them is that made by Rose and Dobson [28], presented in column 5 of Table 1, which, instead of taking slabs, used the semi-infinite jellium system. Those authors took the Ashcroft empty-core pseudopotential [29] and a quasi-local approximation to the response function. Their results show the same trend as ours, although the values are higher.

We note that our perturbative results agree roughly with experimental values. However, the comparison between calculated and experimental surface energies

Table 1

Comparison of LDA surface energies of the most packed $\mathrm{Al}$ faces

\begin{tabular}{|c|c|c|c|c|c|c|c|}
\hline \multirow[t]{3}{*}{ Face } & \multicolumn{3}{|l|}{ Our results } & \multicolumn{3}{|l|}{ Other results } & \multirow[t]{3}{*}{ Experimen } \\
\hline & \multirow{2}{*}{$\frac{\text { Perturbative }}{\text { HA }}$} & \multicolumn{2}{|c|}{ Non-perturbative } & \multirow{2}{*}{$\begin{array}{l}\text { Perturbative } \\
\text { RD }\end{array}$} & \multicolumn{2}{|c|}{ Non-perturbative } & \\
\hline & & AE-i & AE-f & & FCD & PW & \\
\hline$\left(\begin{array}{lll}1 & 1 & 1\end{array}\right)$ & 865 & 1458 & 1272 & 1065 & 1368 & 939 & \\
\hline$\left(\begin{array}{lll}1 & 0 & 0\end{array}\right)$ & 925 & 1546 & 1499 & 1160 & 1520 & 1081 & \\
\hline \multirow[t]{2}{*}{$\left(\begin{array}{lll}1 & 1 & 0\end{array}\right)$} & 1549 & 1699 & 1543 & 1700 & 1459 & 1090 & \\
\hline & & & & & & & 1143,1169 \\
\hline
\end{tabular}

HA denotes second-order perturbative results using HA pseudopotential, and taking the incremental energy method for slabs with 6 and 7 layers. AE-i indicates all-electron calculations using the CRYSTAL98 code and the incremental method (same slabs). AE-f refers the same all-electron calculation, but making a fit to a series of slabs containing 1 to 10 layers. RD denotes Rose and Dobson second-order perturbative results for semi-infinite jellium using the Ashcroft pseudopotential [28]. FCD denotes the full charge density results (we added our estimates of the differences between PBE and LDA to the AE values of Vitos et al. [32]). PW refers to plane-wave calculations of slabs using Hamann-Schluter norm-conserving pseudopotentials $[16,34,35]$. The experimental values, taken from Refs. [30,31], are face independent. All energies are in $\mathrm{erg} / \mathrm{cm}^{2}$. 
must be done with some care. Experimental values, which are obtained for an undifferentiated face are not very reliable. They arose from measurements of the gassolid surface tension, which were extrapolated to zero temperature [30]. Another way to obtain the surface energy uses elastic constants and bulk modulus measurements [31]. Thus, we are led to give more importance to the comparison of the non-perturbative results with good ab-initio results than with experimental data.

Columns 3 (AE-i) and 4 (AE-f) of Table 1 show the results of our fully atomistic calculations with the CRYSTAL98 code. In the first case (AE-i), the surface energies were obtained by the incremental method, using slabs with 6 and 7 layers, while in the second case (AE-f) they were found by the linear fitting method, using slabs containing 1 to 10 layers. In contrast with the perturbative results, non-perturbative ones do not depend so much on the exposed face. Furthermore, our non-perturbative results are higher than perturbative ones.

With the purpose of providing reference values Vitos et al. [32] calculated the surface energies of the same three aluminium faces by the full charge density (FCD) method, which does not require slabs. In Table 4 of [32], they report values of surface energies, which were produced with the GGA-PBE [8] functional instead of the LDA. In Table 2, we compare their values with our own PBE calculations of the same surface energies (not self-consistently performed, but taking LDA densities). The close agreement of these results make us confident of our calculations of aluminium surface energies. Looking at our Tables 1 and 2, we see a systematic energy drop of $12-13 \%$ from LDA to GGA-PBE in our AE calculations. Thus, it is expected that the result of FCD calculations will also be higher in LDA than in GGA-PBE by the same magnitude. Based on this, we estimated the FCD surface energies in the LDA, which are shown in column 6 of Table 1. Our LDA-AE values, obtained by the fitting method, are also close to the FCD values in the same approximation.

Column 7 of Table 1 shows the results of other authors [16,34,35] using plane waves and norm-conserving pseudopotentials to evaluate slab energies. These results, which are very close to experimental data, lie below the FCD ones.

Let us now return to the jelllium model. The stabilized jellium model [36] is a simple extension of the jellium model, whose surface energies are positive for all density parameters. Starting with the surface energy of stabilized jellium at $r_{\mathrm{s}}=2.07$ bohr (952 and $871 \mathrm{erg} / \mathrm{cm}^{2}$ for LDA and GGA-PBE, respectively), we can obtain various surfaces energies multiplying them by convenient corrugation factors, which were calculated by Perdew et al. [33]. In Table 3, we show the resulting surface

Table 2

GGA-PBE surface energies of the most packed Al faces

\begin{tabular}{|c|c|c|}
\hline Face & $\mathrm{AE}$ & $\mathrm{FCD}$ \\
\hline$\left(\begin{array}{lll}1 & 1 & 1\end{array}\right)$ & 1103 & 1199 \\
\hline$\left(\begin{array}{lll}1 & 0 & 0\end{array}\right)$ & 1326 & 1347 \\
\hline$\left(\begin{array}{lll}1 & 1 & 0\end{array}\right)$ & 1355 & 1271 \\
\hline
\end{tabular}

AE denotes all-electron results obtained with CRYSTAL98 and the fitting method for a series of slabs with 1 to 10 layers. FCD denotes results of the full charge-density method by Vitos et al. [32]. All energies are in $\mathrm{erg} / \mathrm{cm}^{2}$. 
energies for LDA and GGA-PBE. The ordering of the three surface energies coincides with our above calculations, either non-perturbative or perturbative. We note the small face anisotropy found by the stabilized jellium model, in agreement with our non-perturbative results.

Let us now examine, in more detail, the incremental and linear fitting methods. The surface energy in the incremental method depends strongly on the considered slab. Fig. 1 shows the variation of the surface energy with the thickness of the slab for our AE calculations.

On the other hand, Fig. 2 shows the variation of the surface energy with respect to the number of slabs included in the fitting procedure. The results appear convergent, so that we may estimate an uncertainty in the surface energy of about 5\% fitting 10 slabs. By comparing Figs. 1 and 2 we conclude that the uncertainty of the surface energy obtained by the fitting method is lower than that obtained by the incremental method.

Table 3

Stabilized jellium surface energies using a convenient corrugation factor [33] for each face

\begin{tabular}{lll}
\hline Face & LDA & PBE \\
\hline$\left(\begin{array}{lll}1 & 1 & 1\end{array}\right)$ & 1095 & 1002 \\
$\left(\begin{array}{lll}1 & 0 & 0\end{array}\right)$ & 1161 & 1063 \\
$\left(\begin{array}{lll}1 & 1 & 0\end{array}\right)$ & 1311 & 1199 \\
\hline
\end{tabular}

All energies are in $\mathrm{erg} / \mathrm{cm}^{2}$.

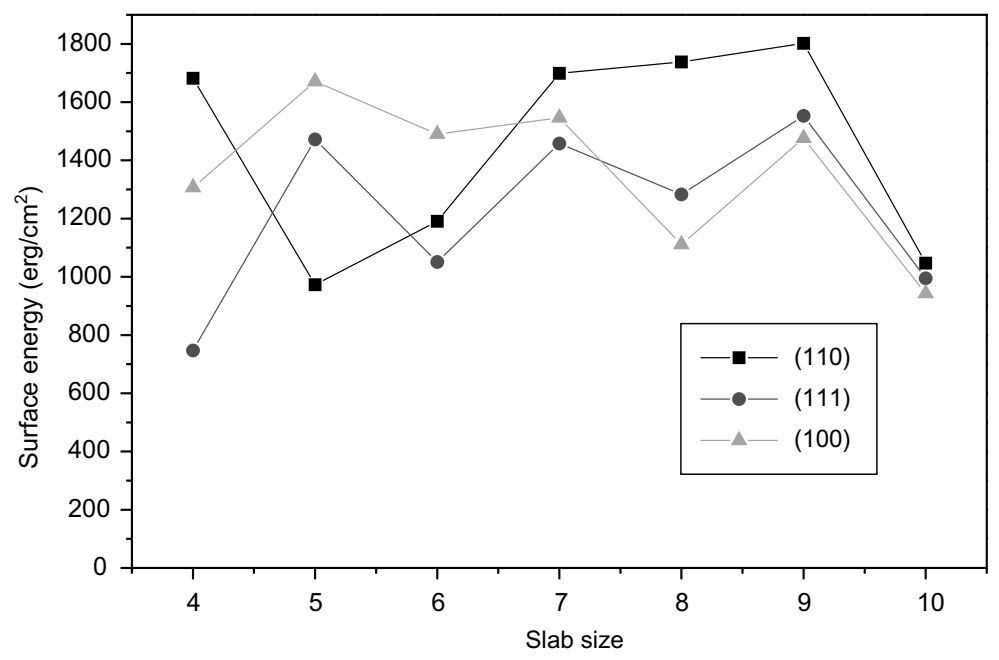

Fig. 1. Dependence of surface energy on number of layers of slabs in the incremental method. Slab energies calculated using all-electron code CRYSTAL98 in the LDA. 


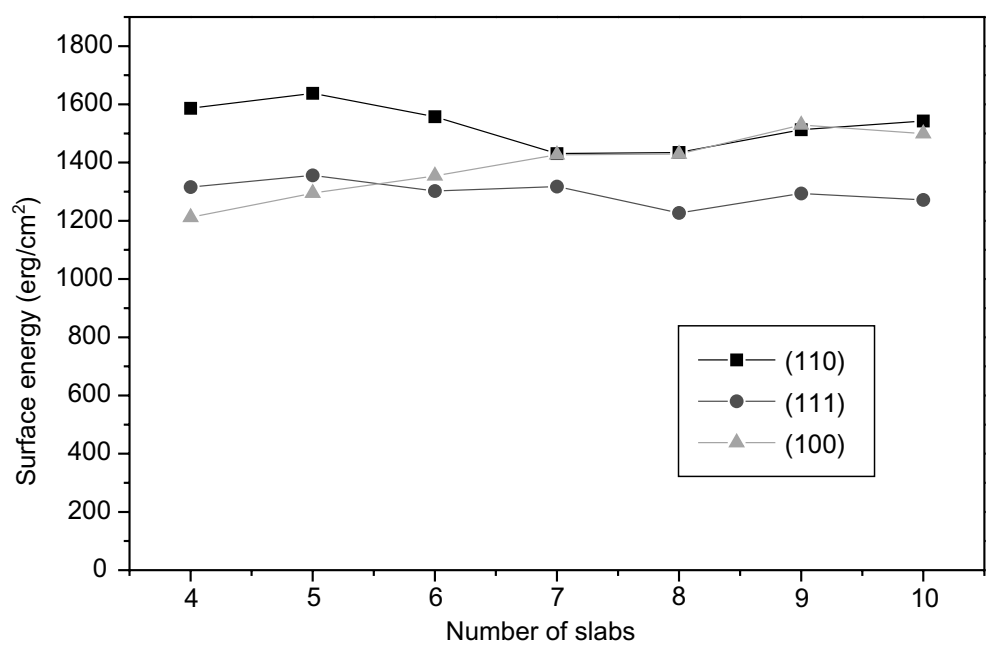

Fig. 2. Dependence of LDA surface energy on number of fitted slabs. Slabs used in fitting procedure are always thickest ones, going from $11-N$ to 10 layers.

\section{Conclusions}

We performed non-perturbative and perturbative calculations of aluminium surface energies. Our perturbative results are similar to those of Rose and Dobson [28], which are one of the very few perturbative surface calculations available. Perturbative values depend more on the considered face than non-perturbative ones. On the other side, better results are obtained from atomistic calculations using Gaussians as basis set. Our values agree with FCD calculations [32], but not with old plane-wave calculations [16,34,35]. The incremental method is a quick way to evaluate the surface energy from slab results, but the fitting method is more reliable, and should be used whenever results for several slabs are available.

\section{Acknowledgements}

We thank J.M. Pitarke and A. Garcia-Lekue (University of Basque Country, Bilbo, Spain) for their collaboration in the perturbative calculations.

\section{References}

[1] J.C. Boettger, Phys. Rev. B 53 (1996) 13133.

[2] J.C. Boettger, Phys. Rev. B 49 (1994) 16798.

[3] V. Fiorentini, M. Methfessel, J. Phys.: Condens. Matter 8 (1996) 6525. 
[4] J.C. Boettger, J.R. Smith, U. Birkenheuer, N. Rsch, S.B. Trickey, J.R. Sabin, S.P. Apell, J. Phys. Condens. Matter 10 (1998) 893.

[5] V. Fiorentini, M. Methfessel, J. Phys.: Condens. Matter 10 (1998) 895.

[6] J.G. Gay, J.R. Smith, R. Richter, F.J. Arlinghaus, R.H. Wagoner, J. Vac. Sci. Technol. A 2 (1984) 931.

[7] W. Kohn, L.J. Sham, Phys. Rev 140A (1965) 1133.

[8] J.P. Perdew, K. Burke, M. Ernzerhof, Phys. Rev. Lett. 77 (1996) 3865.

[9] C. Fiolhais, F. Nogueira, M.A.L. Marques (Eds.), A Primer in Density Functional Theory, Lecture Notes in Physics, Vol. 620, Springer, Berlin (2003).

[10] J.P. Perdew, S. Kurth, A. Zupan, P. Blaha, Phys. Rev. Lett. 82 (1999) 2544.

[11] N.D. Lang, W. Kohn, Phys. Rev. B 1 (1970) 4555.

[12] E. Eguiluz, Phys. Rev. Lett. 51 (1983) 1907.

[13] J.M. Pitarke, A.G. Eguiluz, Phys. Rev. B 57 (1998) 6329; Phys. Rev. B 63 (2001) 45116.

[14] S. Kurth, J.P. Perdew, P. Blaha, Int. J. Quant. Chem. 75 (1999) 889.

[15] L.M. Almeida, J.P. Perdew, C. Fiolhais, Phys. Rev. B 66 (2002) 075115.

[16] K.M. Ho, K.P. Bohnen, Phys. Rev. B. 32 (1985) 3446.

[17] C. Henriques, Ph.D. Thesis, New University of Lisbon, Lisbon, 2002.

[18] C. Fiolhais, C. Henriques, I. Sarria, J.M. Pitarke, Prog. Surf. Sci. 67 (2001) 285.

[19] C. Henriques, A. García-Lekue, I. Sarria, C. Fiolhais, J.M. Pitarke, in preparation.

[20] V. Heine, I. Abarenkov, Philos. Mag. 9 (1964) 451.

[21] I. Abarenkov, V. Heine, Philos. Mag. 12 (1965) 529.

[22] F. Nogueira, C. Fiolhais, J.P. Perdew, Phys. Rev. B 59 (1999) 2570.

[23] C. Pisani (Ed.), Quantum-Mechanical Ab-initio Calculation of the Properties of Crystalline Materials, Lecture Notes in Chemistry, Vol. 67, Springer, Berlin, 1996.

[24] E. Eguiluz, Phys. Rev. B 35 (1987) 5473.

[25] J.P. Perdew, Y. Wang, Phys. Rev. B 45 (1992) 13244.

[26] V.R. Saunders, R. Dovesi, C. Roetti, M. Causà, N.M. Harrison, R. Orlando, C.M. Zicovich-Wilson, CRYSTAL98 User's Manual, University of Torino, Torino 1998.

[27] L.M. Almeida, C. Fiolhais, M. Causà, Int. J. Quant. Chem. 91 (2003) 224.

[28] J.H. Rose, J.F. Dobson, Solid State Commun. 37 (1981) 91.

[29] N.W. Ashcroft, Phys. Lett. 23 (1966) 48.

[30] W.R. Tyson, W.A. Miller, Surf. Sci. 62 (1977) 267.

[31] H. Wawra, Z. Metallkd. 66 (1975) 395.

[32] L. Vitos, A.V. Ruban, H.L. Skriver, J. Kollár, Surf. Sci. 411 (1998) 186.

[33] J.P. Perdew, Y. Wang, E. Engel, Phys. Rev. Lett. 66 (1991) 508.

[34] K.P. Bohnen, K.M. Ho, Surf. Sci. 207 (1995) 105.

[35] J. Schöchlin, K.P. Bohnen, K.M. Ho, Surf. Sci. 324 (1995) 113.

[36] J.P. Perdew, H.Q. Tran, E.D. Smith, Phys. Rev. B 42 (1990) 11627. 